\title{
Study of the Plasma near the Plasma Electrode by Probes and Photodetachment in ECR-driven Negative Ion Source
}

\author{
M. Bacal ${ }^{\mathrm{a}}$, P. Svarnas ${ }^{\mathrm{a}}$, S. Béchu ${ }^{\mathrm{b}}$, J. Pelletier ${ }^{\mathrm{b}}$ \\ ${ }^{a}$ LPTP, UMR CNRS 7648, Ecole Polytechnique, 91128 Palaiseau, France \\ ${ }^{\mathrm{b}}$ Laboratoire de Physique Subatomique et de Cosmologie, UMR CNRS 5821, \\ 53 rue des Martyrs, 38026 Grenoble, France
}

\begin{abstract}
The effect of the plasma electrode bias on the plasma characteristics near the extraction aperture in a large volume hybrid multicusp negative ion source, driven by $2.45 \mathrm{GHz}$ microwaves, is reported. Spatially resolved negative ion and electron density measurements were performed under various pressures (1-4 mTorr) by means of electrostatic probe and photodetachment technique.
\end{abstract}

Keywords: Negative ions, plasma electrode bias, magnetic filter, extracted beams

PACS : 32.80.Gc, 52.27.Cm, 29.25.Ni, 52.80.Pi

\section{INTRODUCTION}

Contemporary negative ion sources operate with a magnetic filter field extending up to the plasma electrode (PE) which contains the extraction aperture ${ }^{1}$. It is generally accepted that a positive PE bias $\left(V_{P E}\right)$ reduces the extracted electron current. In some experiments, an optimum $V_{P E}$ for negative ion extraction was found ${ }^{2-4}$. A possible cause of these effects was suggested in ${ }^{3}$ : electrons are trapped in the weak magnetic field and lost along the field lines, whereas positive and negative ions are not trapped because of their large Larmor radius. More negative ions arrive from the bulk plasma to ensure plasma neutrality. However, a definite explanation of the $\mathrm{H}^{-}$ion current peak, when the PE bias is varied, is still not available.

The electrostatic particle simulation ${ }^{1,5,6}$ without applied PE bias showed that the presence of the weak magnetic field produces important modifications in the positive ion flow and as a result in the structure of the plasma potential. A characteristic peak in the plasma potential $\left(V_{p}\right)$ dependence on the distance from the PE $(D)$ is predicted, where many negative ions are localized.

The purpose of this work is the experimental study of the spatial distribution of the plasma parameters in the vicinity of the PE, specifically on the axis of the extraction aperture, using electrostatic probes and laser photodetachment.

\section{EXPERIMENTAL SETUP and DIAGNOSTICS}

The $\mathrm{H}^{-}$ion source Camembert III has been described in ${ }^{4}$ and a draft is shown in Fig. 1 . The magnetic multipole chamber Camembert III was previously equipped with 

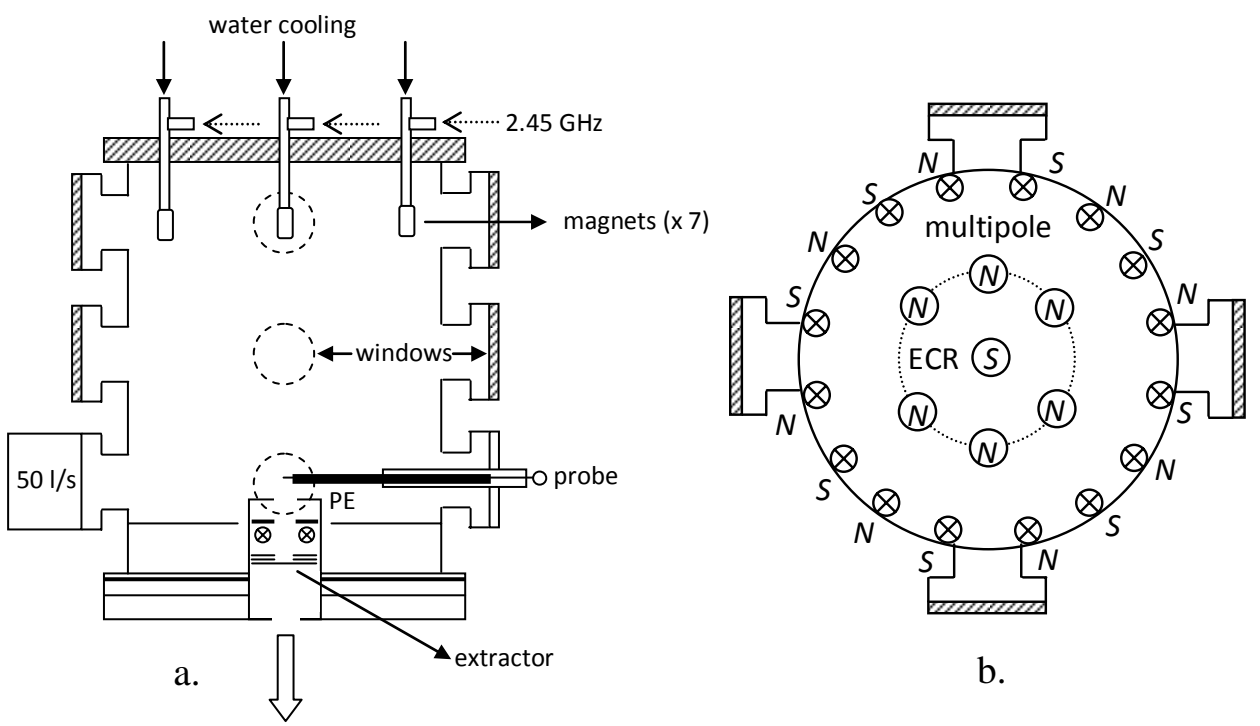

b.

FIGURE 1. Schematic representation of the ECR-driven hybrid multucusp source Camembert III (a) and the magnets forming the multicusp configuration (b).

16 filaments, installed on the top flange. It was operating in a dc mode, similar to a filamented ion source. Since 2003, a 2-D network of seven elementary independent ECR plasma sources ${ }^{7}$ has been installed on the top flange. They are powered by microwaves $(2.45 \mathrm{GHz})$ at $0.9 \mathrm{~kW}$, equally distributed at the seven elementary sources. Each source contains a permanent magnet which provides the magnetic field required for ECR (875 G). This magnetic field also confines the fast electrons. It represents a first magnetic filter and the region around the magnet represents the “driven region".

Opposite to the top flange there are the PE with the extraction hole, followed by the extraction electrode equipped with a pair of magnets for electron separation, and the acceleration electrodes. The stray magnetic field from the magnets located in the extraction electrode extends into the plasma and provides a second magnetic filter. This magnetic field attains a maximum of $20 \mathrm{G}$ at a distance of $1.6 \mathrm{~cm}$ from the PE. This region can be denoted as the "extraction region". A large volume of cold plasma separates the above two extreme regions.

The various plasma parameters were measured with an electrostatic probe made of tungsten wire of $0.5 \mathrm{~mm}$ diameter, mounted on a three-axis manipulator installed on a flange at the PE level. A tip of $15 \mathrm{~mm}$ was exposed to the plasma, while the rest was shielded by a ceramic cylinder. This probe was L-shaped so that its longer part was coaxial with the laser beam, used for measuring the negative ion density by photodetachment ${ }^{8}$. The measurements were effected on the axis passing through the center of the extraction opening.

A Nd-YAG laser (repetition rate $10 \mathrm{~Hz}$, photon energy $1.2 \mathrm{eV}$ ) was used to illuminate the plasma, through a $6 \mathrm{~mm}$ diaphragm, and the photodetached electrons from the $\mathrm{H}^{-}$ions were collected by the L-shaped, positively biased, probe. The probe transient current, generated by the collected photodetached electrons, was recorded on a digital oscilloscope (500 MHz, 2 GSample/s) operating in averaging mode, via 
capacitive decoupling. Since previous studies showed that in the region near the PE the negative-ion relative density $n_{H}{ }^{-} / n_{e}$ is much higher than in the center of the source, the analysis of the probe signals was carried out according to the theory and recommendations of ${ }^{9}$.

\section{SPATIAL VARIATION of the PLASMA PARAMETERS}

The results obtained at a pressure $p$ of 3 mTorr are presented in Fig. 2 to 6. The electron density spatial variation is shown in Figure 2. The electron density goes down linearly with the distance to the PE. The higher the positive bias $V_{P E}$, the lower the electron density. This shows that the electrons are efficiently swept away from the PE neighborhood due to the applied positive PE bias.

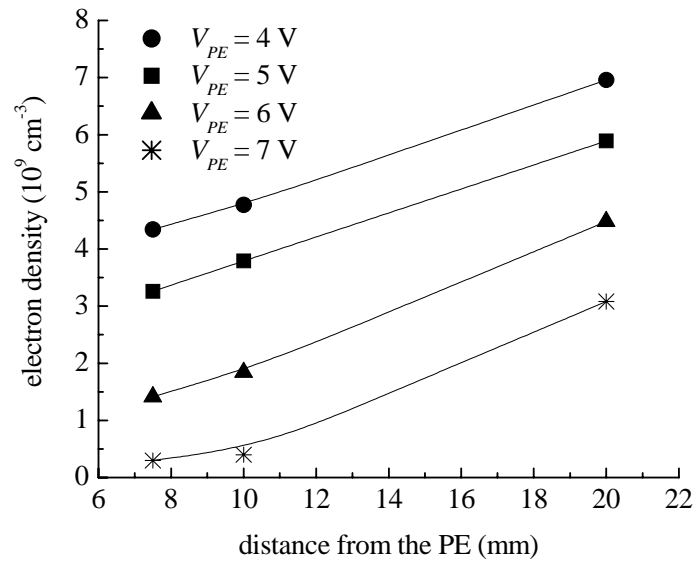

FIGURE 2. Variation of electron density with distance from the plasma electrode, for four values of the plasma electrode bias $V_{P E}$.

The spatial variation of the negative ion density (Fig. 3) clearly indicates its enhancement at a distance close to the plasma electrode where the electron density tends to be reduced. The optimum PE bias is $V_{P E}=5.5 \mathrm{~V}$.

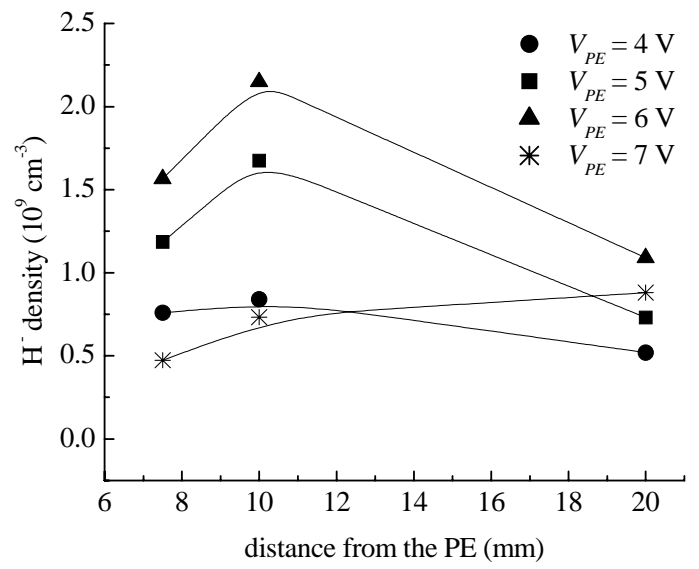

FIGURE 3. Variation of the negative ion density with distance from the plasma electrode, for four values of the plasma electrode bias $V_{P E}$. 
Fig. 4 shows the variation of the relative negative ion density $n_{H}{ }^{-} / n_{e}$ versus $D$. Note that this relative density goes up with the PE bias and attains the value of 1.9 for the highest $V_{P E}$.

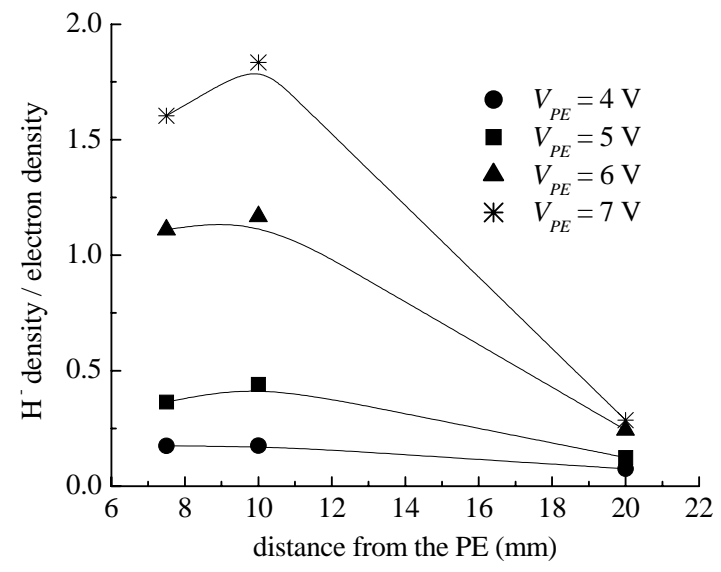

FIGURE 4. Variation of the relative negative ion density with distance from the plasma electrode, for four values of the plasma electrode bias $V_{P E}$.

An indication of a weak plasma potential peak versus $D$ exists for $V_{P E}>4 \mathrm{~V}$. This "maximum" is located at a distance of $1 \mathrm{~cm}$ (Fig. 5).

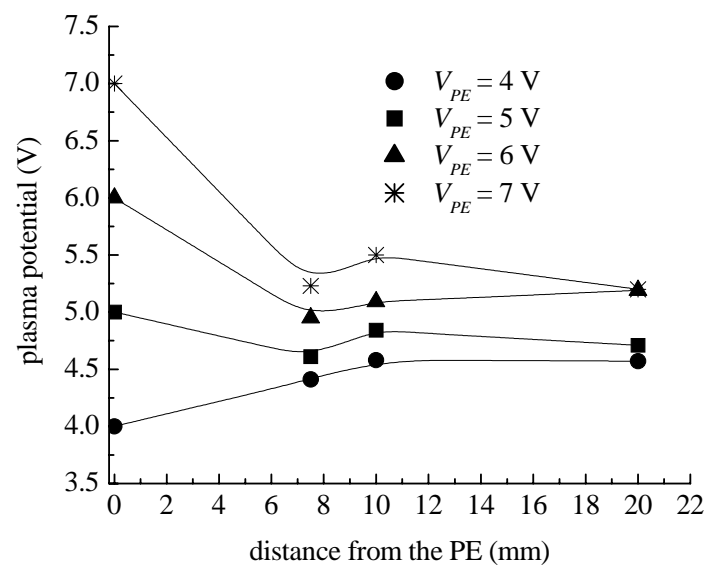

FIGURE 5. Variation of the plasma potential with distance from the plasma electrode, for four values of the plasma electrode bias $V_{P E}$.

The spatial variation of the electron temperature (Fig. 6) indicates that for $V_{P E}<6 \mathrm{~V}$ the electron temperature is low $(0.3-0.5 \mathrm{eV})$ at all the distances studied, but goes up abruptly near the PE when $V_{P E}>6 \mathrm{~V}$. 


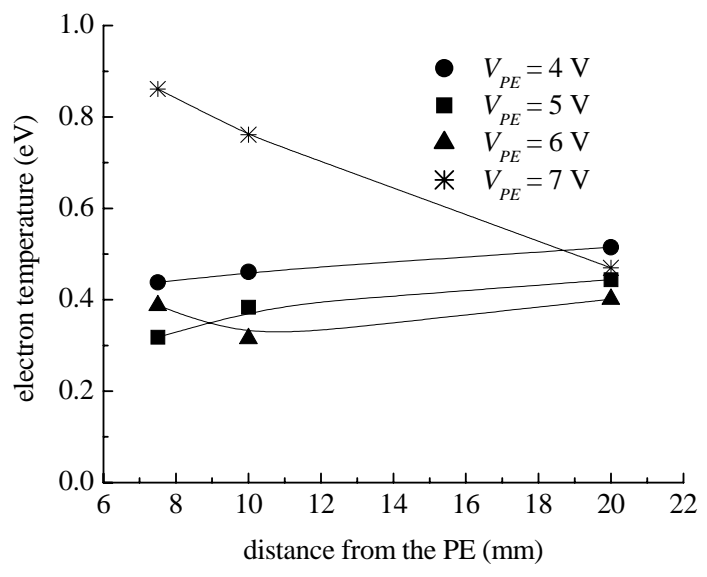

FIGURE 6. Variation of the electron temperature with distance from the plasma electrode, for four values of the plasma electrode bias $V_{P E}$.

A comparison on the pressure dependence of the negative ion densities at $150 \mathrm{~mm}$ and $7.5 \mathrm{~mm}$ from the PE shows that the latter is enhanced particularly at the lowest pressure (Fig. 7). Even at a distance of $20 \mathrm{~mm}$ the effect of the magnetic field and the PE bias starts to go down, as can be seen in Fig. 3.

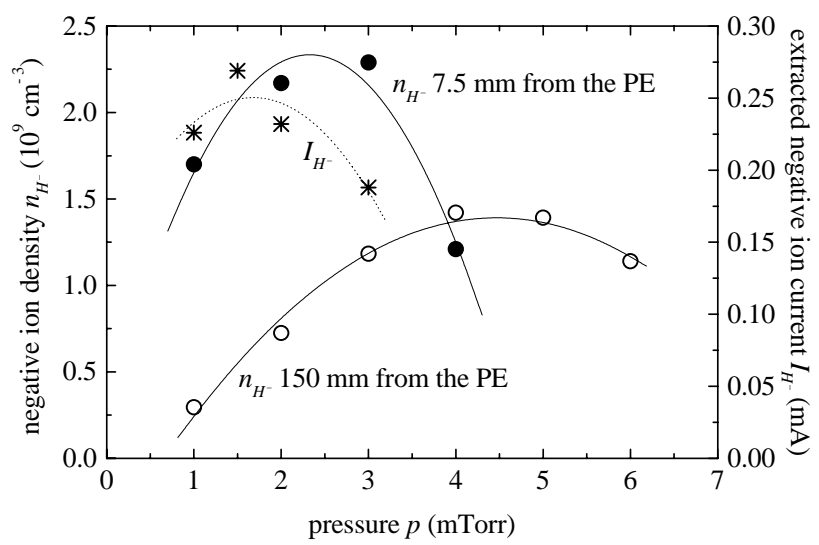

FIGURE 7. Comparison between the negative ion density close to the extractor aperture (solid symbols) and at the center of the source (open symbols) vs. pressure. The extracted negative ion current is also compared (dotted line). ${ }^{4}$

The extracted negative ion current $I_{H^{-}}$is correlated better with the plasma parameters near the extraction opening (Fig. 7) than with those in the center of the source (150 $\mathrm{mm}$ from the PE). The optimum pressure at the center of the source lies between 4 and 5 mTorr, as opposed to $1.5-3$ mTorr at $7.5 \mathrm{~mm}$ from the PE. The latter approaches the optimum pressure for the extracted $\mathrm{H}^{-}$ion current.

Fig. 8 shows the ratio $n_{H}{ }^{-} / n_{e}$ between the negative-ion density and the electron density found at a distance of $7.5 \mathrm{~mm}$ from the PE when $V_{P E}$ is varied. In the same figure the ratio $I_{H^{-}} / I_{e}$ of the extracted currents is as well presented. 


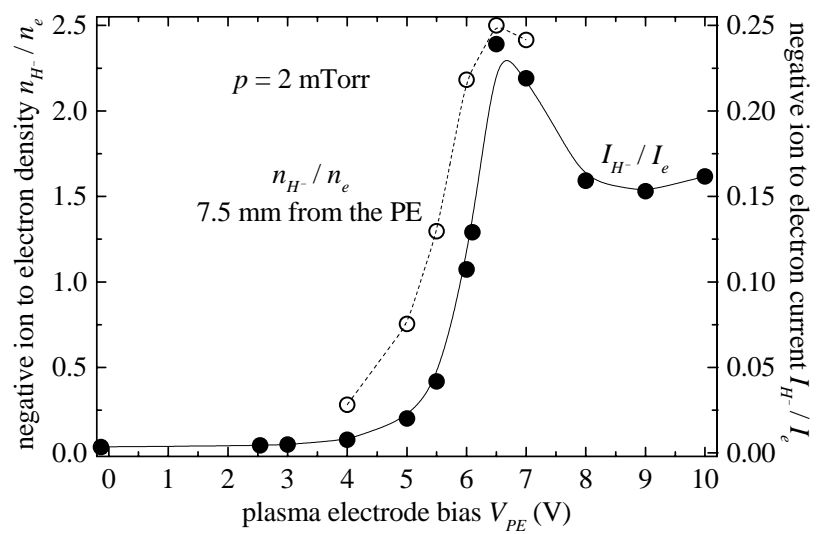

FIGURE 9. Ratios of the negative ion to electron density (dashed line) and the extracted negative ion to electron current (solid line) vs. plasma electrode bias. ${ }^{4}$

\section{CONCLUSION}

Compared to the electron density at the center of the source $\left(3 \times 10^{10} \mathrm{~cm}^{-3}\right.$ at $3 \mathrm{mTorr}$ ), the electron density at $1 \mathrm{~cm}$ from the plasma electrode is reduced by a factor 30, when a PE bias $V_{P E}=6 \mathrm{~V}$ is applied.

The plasma potential varies abruptly near the PE for extreme bias values, when ion or electron sheaths are formed, but stays approximately constant for the medium value $V_{P E}=5.5 \mathrm{~V}$, which is close to the plasma potential far from the PE. Let us denote this value $V_{P E}{ }^{*}$. This value of the $\mathrm{PE}$ bias - $V_{P E}{ }^{*}$ - is important since the maximum negative ion density occurs at this bias (not shown in Fig. 3). The electron temperature values increase abruptly when $V_{P E}>V_{P E}{ }^{*}$ (see Fig. 6).

The highest negative ion densities are found in the neighborhood of the plasma electrode, at distances where the electron density is reduced.

Note that the ratio $n_{H}{ }^{-} / n_{e}$ attains the value 1.9 (Fig. 4), which means that the negative ions constitute the majority of the negative species. We should remind that in the filamented version of Camembert III values of $n_{H}{ }^{-} / n_{e}$ as high as 12 were measured in a discharge of $2.5 \mathrm{~kW}$. It was observed that the maximum $n_{H}{ }^{-} / n_{e}$ ratio went up when the plasma density or discharge power was enhanced. This may explain the lower $n_{H}{ }^{-} / n_{e}$ value (i.e. $\sim 2$ ) found in this, ECR driven, version of CAMEMBERT III, where the plasma density is limited by the available microwave power.

In conclusion the explanation of the $\mathrm{H}^{-}$ion density peak can be summarized as follows ${ }^{4}$ : the depletion of the electron population, when the positive bias of the PE is enhanced, disturbs the local plasma neutrality and new negatively charged particles should replace the electrons collected by the PE. In a collisionless situation, the electrons present in the bulk plasma are unable to cross the transverse magnetic field in front of the PE. Therefore, negative ions from the central plasma replace the depleted electrons in the magnetized region, as suggested in ${ }^{3}$. Indeed, the measured $\mathrm{H}^{-}$ ion density increases close to the PE and peaks when $V_{P E}$ is slightly greater than $V_{P E}{ }^{*}$. This results in higher $\mathrm{H}^{-}$ion extracted current. When the PE bias is further increased the $\mathrm{H}^{-}$ions are accelerated toward the PE. The negative-ion residence time decreases 
due to the larger velocity and the $\mathrm{H}^{-}$density near the $\mathrm{PE}$ as well as the extracted negative-ion current go down.

\section{REFERENCES}

1. M. Bacal, A. Hatayama, J. Peters, IEEE Trans. Plasma Sci., 33, № 6, 2005, 1845

2. K.N. Leung, K.W. E.hlers, M. Bacal, Rev. Sci. Instrum., 54, 1983, 56

3. M. Bacal, J. Bruneteau, P. Devynck, Rev. Sci. Instrum., 59, 1988, 2152

4. P. Svarnas, J. Breton, M. Bacal, R. Faulkner, IEEE Trans. Plasma Sci., 35, N4, 2007, 1156

5. T. Sakurabayashi, A. Hatayama, M. Bacal, A.I.P. Conference Proceedings 763, Editors J.D. Sherman, Y.I. Belchenko, 2005, 96

6. A. Hatayama, Rev. Sci. Instrum. 79, 2008, 02B901

7. A. Lacoste, T. Lagarde, S. Béchu, Y. Arnal, J. Pelletier, Plasma Sources Sci. \& Technol., 11, 2002, 407

8. M. Bacal, Rev. Sci. Instrum., 71, 2000, 3981

9. F.El Balghiti-Sube, F.G. Baksht, M. Bacal, Rev. Sci. Instrum., 67, 1996, 2221 